\title{
Cost-efficient composite processing techniques for aerospace applications - a review
}

\begin{abstract}
Composite materials have been used in aircraft components since the early beginning of aircraft industry establishment.Undenaible advantages of composites in mechanical properties and light weight in comparison with conventional metal alloys make them desirable alternative for fabrication of different aircraft components. However, quality concerns and high costs of processing tackle the extensive usage of composites in aircraft structure, until the past decade, introducing new generation of composite processing techniques, needless of traditional autoclave processing and capable of fabricating aerospacegrade quality composite parts more time and cost efficiently. In this paper concise review over recent cost-efficient composite processing technologies with proven practicality in commercial aircraft applications, is presented.
\end{abstract}

Keyword: Composite processing; Out-of-autoclave; Aircraft components; Cost-efficient process 